\title{
SLOW FEEDBACK LOOPS FOR A LANDAU CAVITY WITH HIGH BEAM LOADING
}

\author{
N. Towne*, National Synchrotron Light Source \\ Brookhaven National Laboratory, Bldg. 725B, Upton, NY 11973-5000 ${ }^{\dagger}$
}

\begin{abstract}
Equilibrium bunch shapes for bunches stretched with a higher-harmonic cavity are sensitive functions of beam and RF parameters. This paper gives results of calculations of equilibrium bunch shapes as a function of Landau-cavity phase, the relationship between the stretched-beam phase and slow feedback controls for the cavity is described, and a control system capable of stretching, compressing, controlling the cavity phase arbitrarily (within the limits of the power amplifier), and operating without beam for use in the NSLS Vacuum-Ultraviolet (VUV) ring is presented. The last is compared with the existing RF controls for the VUVring harmonic cavity [1].
\end{abstract}

\section{INTRODUCTION}

The sensitivity of the bunch shapes to the RF-system parameters of a Landau cavity has important implications to the RF controls for the cavity. Small shifts in the bunch's potential well shift the bunch centroid by an exaggerated amount through changes in the equilibrium bunch distribution. In particular, shifts in the phase of the harmonic cavity voltage have an amplified effect on the beam phase. There is negative feedback, when there is beam loading, resulting from the movement of the bunch centroid. This is described in section 2 .

The original control system for the powered harmonic cavity, the one that is in use now [1], has worked remarkably well with very high beam loading. There are three loops - the tuning loop, a loop that levels the forward power on the transmission line, and a loop that levels the cavity field by controlling the phase of the forward power. The tuning loop controls the phase of the cavity field relative to the forward wave on the transmission line [2]. The crossed level and phase loops work well with substantial beam loading because of the large detuning of the cavity. (By crossed loops is meant that the phase detector in one loop drives the attenuator and the level detector drives the phase shifter in the drive to the amplifier.) The shortcoming of the existing system is that it is not able to vary the phase of the cavity over a sufficiently wide range due to the polar nature of the RF modulators and the fact that the cavity tuning is controlled through the incident RF wave on the transmission line. For this reason bunch shapes are not optimal.

\footnotetext{
*Email: towne@bnl.gov

$\dagger$ Work performed under the auspices of the U.S. Department of Energy, under contract DE-AC02-76CH00016.
}

Section 3 contains a description of a proposed RF control system capable of stretching, compressing, controlling the cavity phase arbitrarily (within the limits of the power amplifier in high beam loading), and operating without beam. In that section it is assumed that the main-cavity field is fixed and that the harmonic-cavity loops are slow compared to the coherent frequencies: coherent instabilities [3] (aside from the equilibrium-phase instability) are not considered.

Machine parameters are given in table 1.

Table 1: Values of VUV ring, cavity parameters, and symbols.

\begin{tabular}{|l|r|l|}
\hline Beam energy & $E_{0}$ & $800 \mathrm{MeV}$ \\
Energy loss per turn & $U_{0}$ & $20.4 \mathrm{keV}$ \\
Momentum compaction & $\alpha$ & 0.0245 \\
Revolution frequency & $\omega_{0} / 2 \pi$ & $5.8763 \mathrm{MHz}$ \\
RF peak voltages & $V_{1} / V_{2}$ & $80 / 20 \mathrm{kV}$ \\
RF harmonic numbers & $h_{1} / h_{2}$ & $9 / 36$ \\
\hline
\end{tabular}

\section{BUNCH SHAPES}

This section considers the sensitivity of stretched bunch shapes to cavity phase. Due to the use of a near- $\phi^{4}$ potential, bunch shapes [4] are sensitive functions of the harmonic cavity field - in particular the phase-when the bunches are optimally stretched. This occurs because the equilibrium distribution sloshes in the shallow potential well. Furthermore, the shifts of the beam phase and the cavity phase are of opposite signs. To see this, if the harmonic-cavity phase is shifted so that the harmoniccavity voltage lags a small amount, the total voltage is shifted downward locally. This shift moves the synchronous phase forward in time to where the main cavity, having a lower frequency, brings the waveform up. In this way the new synchronous phase leads the original synchronous phase. The amount by which it leads is large compared to the original phase shift since the RF waveform is locally flat. The degree to which the bunch centroid moves depends on the intrinsic energy spread. In figure 1 is shown calculated bunches shapes for the VUV ring when optimally stretched and when the cavity phase has been shifted by $-2^{\circ}$. The bunch phase is shifted by the factor -4.5 times the cavity-phase shift.

This phase shift of the beam in response to a perturbation of the cavity phase, through beam loading, provides negative feedback from the beam to the cavity field. This negative feedback is a mechanism by which perturbations of the cavity phase are suppressed. This is to say, e.g., that 


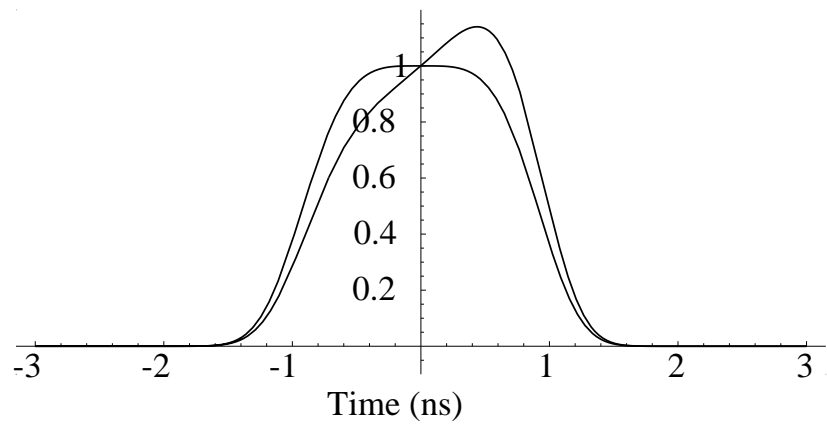

Figure 1: Calculated bunches shapes for the VUV ring when optimally stretched and when the cavity phase has been shifted by $-2^{\circ}$. In the second case the beam phase has shifted $+9^{\circ}$ at the harmonic-cavity frequency.

a perturbation of the generator current affecting the phase of the total current is largely cancelled by the shift in the beam's contribution to the total current in the cavity. An estimate of the degree to which this cancellation occurs, in the limit that $\left|I_{g}\right| \ll\left|I_{b}\right|$ and $I_{g}$ is approximately orthogonal to $I_{T}$, goes as follows. One expresses the perturbation in the phase of the cavity voltage $\delta \Psi_{V}$ as

$$
\begin{aligned}
\delta \Psi_{V} & =\delta \Psi_{I_{T}} \\
& \simeq \delta \Psi_{I_{b}}+\delta \Psi_{V 0} \\
& \simeq G \delta \Psi_{v}+\delta \Psi_{V 0}
\end{aligned}
$$

where $G$ is the phase multiplication factor obtained from figure $1(-4.5)$ and $\delta \Psi_{V_{0}}$ is a shift in the phase of the cavity field that would be present in the absence of a shift in the beam phase. Solving this last equation for $\delta \Psi_{V}$, we have

$$
\delta \Psi_{V} \simeq \frac{1}{1-G} \times \Psi_{V 0}
$$

In summary, the shift in the bunch centroid due to perturbations influencing the harmonic-cavity phase reduces such perturbations by the factor $1 /(1-G)$.

A consequence of this result is that much larger swings of amplifier power are required to vary the cavity phase a given amount than if there were no shift in the beam phase. In fact, one would like that the forward-wave amplitude on the transmission line be able to pass through zero to different quadrants of the forward-wave plane. This is inconsistent with features of the existing control system.

Therefore, conclusions to be carried to the next section are that:

- control of the cavity tuning using the difference between phases of the cavity and the forward wave on the transmission line is not adequate and

- a vector modulator must be used in place of a phase shifter/attenuator combination for the control of the drive to the power amplifier.

Both conclusions are necessitated by the fact that the forward-wave amplitude must be able to go through zero.

\section{HARMONIC CAVITY CONTROLS}

In this section is discussed the proposed control system for the harmonic cavity. Several major functions the system is required to perform are:

- stretched-bunch operation,

- compressed-bunch operation,

- passive operation for injection, and

- off-line or low-current operation.

In stretched-bunch mode the control system must be able to control the cavity phase over a substantial range. This range is limited by the RF power available.

In figure 2 is shown the complete configuration for the control system proposed here. In the figure a complexphase modulator (CPM) is used to control the drive to the amplifier. The reason for this, as discussed in section 2, is that control through cartesian coordinates is more appropriate for this problem where the forward-wave amplitude $a$ on the transmission line may be required to go near zero and into different quadrants of the complex- $a$ plane.

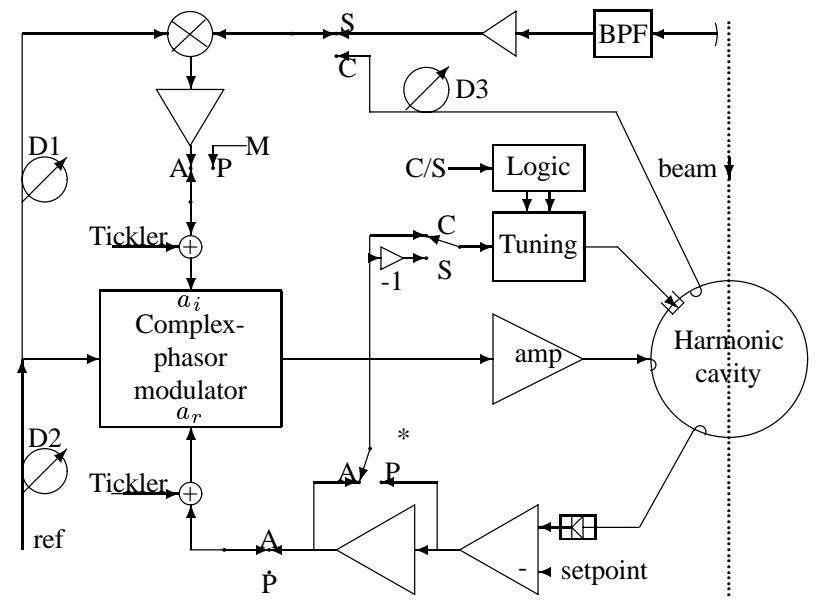

Figure 2: Configuration for the harmonic-cavity control system. The labels ' $\mathrm{A}$ ' and ' $\mathrm{P}$ ' represent switch positions for active and passive operating modes respectively and ' $\mathrm{S}$ ' and ' $C$ ' represent switch positions for stretched and compressed operating modes. The ticker inputs are intended for diagnostic use only. The active/passive switch marked with the asterisk selects a lower loop gain for the passive mode.

For the level regulation of the cavity ordinary envelop detection is used to sense the cavity voltage and provide feedback to $a_{r}$ (the real part of $a$; the nominal beam phase provides the phase reference so that $a_{r}$ is in-phase with the beam while $a_{i}$ is in quadrature with the beam); phase detection is through a mixer and the phase of the beam is used to generate the phase error instead of the phase of the cavity. There are two reasons for doing this. The first is that the bunch-phase factor $G$ provides additional gain compensating the $1 /(1-G)$ factor of equation 4 . The second is that the beam phase is the quantity that needs to be regulated; it is the most appropriate diagnostic for maintaining optimal bunch stretching. 
Cavity detuning is handled differently than in the usual method of detuning for beam-loading compensation [5] where the tuning error signal is derived from the phase between the cavity field and the forward wave on the transmission linearg $V / a$. The latter method is not applicable here because it is required that tuning control operate when the forward wave is zero. To resolve this problem the tuning block of figure 2 is required to move the cavity resonant frequency upwards for a given sign of $a_{r}$ and limit the detuning to the high side of the RF frequency. In this way the modulation amplitude $a_{r}$ is kept at zero, even when $a_{i}$ is small.

When using the harmonic cavity to compress the bunch, the phase of the cavity is reversed so that the slope of the RF wave at the phase of the bunch is increased. This is in contrast to the stretched-bunch mode where the harmonic cavity cancels the slope in the RF wave generated by the main cavity. There are two main differences in the lowlevel controls between these two modes. The first is that the cavity phase is used as feedback instead of the beam phase because the gain advantage of beam feedback in stretched operation is not present in compressed operation. The other difference is that the tuning control is required to move the resonant frequency in the opposite direction-to the low side of the RF frequency-and to confine it there. The -1gain and tuning-logic blocks of figure 2 make this happen.

In passive operation the RF amplifier and CPM are switched off and the cavity driven by beam alone. In this configuration the tuning servo loop, which is used during active operation, may be used to level the RF field in the cavity by detuning the cavity sufficiently, when there is sufficient beam current, to provide the correct field in the cavity. The starred active/passive switch of figure 2 adjusts the gain of the loop appropriately. Tuning control is as described for powered (active) operation. Passive operation is used during injection. Alternatively, one can dispense with this loop altogether by using fixed detuning.

The transition from passive to active operation occurs as follows. After the ring is filled and ramped and the harmonic-cavity control systems is switched to active, there is a jump in the operating point of the CPM that brings the cavity phase to the optimum for stretching (when stretching). In this state $a_{i}$ jumps to a new value and $a_{r}$ becomes nonzero. In response to the latter condition the cavity tuning shifts to bring $a_{r}$ to zero. When this is completed the CPM is at its normal active operating levels.

Off-line operation with cavity voltage leveled is not accommodated explicitly in figure 2 and is accomplished by fixing the detuning of the cavity. The level-regulating loop controlling $a_{r}$ provides the drive to the CPM. Open-loop operation requires opening the level-regulating loop and applying a bias to terminal $\mathrm{M}$ or one of the 'tickler' inputs.

\section{CONCLUSIONS}

This control system described here, intended to be slow compared to the coherent motion, provides passive and ac- tive operation for both stretched and compressed modes. Arbitrary cavity phases can be maintained, within the limit of available RF power, and cavity tuning does not require use of the forward wave on the transmission line. Beam phase has the best potential for maintaining optimal bunching and is used for phase-loop feedback in stretched mode; in compressed mode the cavity phase is used. The crosscoupled amplitude and phase control loops present in the existing control system are retained.

Perturbations of the cavity phase are reduced by negative feedback provided by the beam sloshing in a flat potential well. The implications of this fact on the cavity control system are explored.

The control system described here is far from optimal in the context of what the formalism of optimal-control theory offers to the control of RF systems [6]. Optimal control theory requires, when there is beam loading, that a realistic model of the beam be available or nonsensical results are obtained. Unfortunately, theoretical models of the behavior of stretched beams are not available and one must rely on the use of empirically constructed models. Development of such empirical models is a challenge and is a longer-term prospect at NSLS.

\section{REFERENCES}

[1] R. Biscardi, S. L. Kramer, and G. Ramirez, Nucl. Instrum. Methods A 366 (1995), p. 26-30.

[2] F. Pedersen, IEEE Trans. Nuc. Sci., Vol. NS-32 No. 5, p. 2138 (1985).

[3] S. Krinsky and J. M. Wang, Particle Accelerators Vol. 17, p. 109. (1985)

[4] M. Sands, SLAC Report No. SLAC-0121 (UC, Santa Cruz), Nov 1970.

[5] W. Broom, J. M. Wang, BNL Report 62789 (1996).

[6] D. Boussard and E. Onillon, CERN Report No. CERN SL/9309 (RES), (1993). 